\title{
Comparative study of vitrectomy combined with internal limiting membrane peeling and vitrectomy combined with internal limiting membrane flap covering in idiopathic macular hole treatment: a meta-analysis and systematic review
}

\author{
Tao Jiang ${ }^{1,2}$, Lingmin Zhang ${ }^{3}$, Qi Wan ${ }^{1,2}$, Zhiyong Zhang ${ }^{1,2}$ \\ ${ }^{1}$ The Second Clinical Medical College of Zhejiang Chinese Medical University, Hangzhou, China; ${ }^{2}$ Department of Ophthalmology, The Second \\ Affiliated Hospital, Zhejiang University School of Medicine, Hangzhou, China; ${ }^{3}$ Department of Ophthalmology, Ningbo Eye Hospital, Ningbo, \\ China \\ Contributions: (I) Conception and design: T Jiang, Z Zhang; (II) Administrative support: Z Zhang; (III) Provision of study materials or patients: T \\ Jiang, L Zhang; (IV) Collection and assembly of data: T Jiang, L Zhang; (V) Data analysis and interpretation: T Jiang, L Zhang, Q Wan, Z Zhang; (VI) \\ Manuscript writing: All authors; (VII) Final approval of manuscript: All authors. \\ Correspondence to: Zhiyong Zhang. The Second Clinical Medical College of Zhejiang Chinese Medical University, Hangzhou 310053, China; \\ Department of Ophthalmology, The Second Affiliated Hospital, Zhejiang University School of Medicine, Hangzhou 310009, China. \\ Email: zhangziyongnet@163.com.
}

Background To compare the therapeutic effects of vitrectomy (PPV) combined with the internal limiting membrane (ILM) flap coverage and PPV in combination with ILM peeling on the idiopathic large macular hole $(\mathrm{MH})$, in order to better guide the treatment of large $\mathrm{MH}$.

Methods: Searching was conducted within PubMed, Web of Science, Embase, CNKI, and Wanfang databases, and relevant pieces of literature between 2010 and 2020 published in English or Chinese were included.

Results: A total of 11 studies including 667 patients and 667 affected eyes were included; the effective rate of hole closure between the 2 groups were compared in 11 studies. Results exhibited $94.4 \%$ (286/303 eyes) in the test group (PPV combined with ILM flap coverage) and 85.8\% (313/364 eyes) in the control group (PPV combined with ILM peeling) were closed. $\mathrm{MH}$ closure rates in the test group was superior to the control group [odds ratio $(\mathrm{OR})=3.36,95 \%$ confidence interval $(\mathrm{CI}): 1.88-6.01, \mathrm{P}<0.001$ ]. All 11 studies compared the preoperative and postoperative best corrected visual acuity (BCVA), with no significant difference in the preoperative test control group [standardized mean difference (SMD) $=-0.18,95 \% \mathrm{CI}:-0.42$ to 0.06 , $\mathrm{P}=0.149]$. The BCVA after surgery was better in the test group compared with the control group (SMD $=-0.91,95 \% \mathrm{CI}:-1.43$ to -0.40$), \mathrm{P}=0.001)$.

Discussion: Compared with PPV combined with ILM peeling, PPV combined with ILM flap coverage can significantly improve the $\mathrm{MH}$ closure rate and postoperative BCVA.

Keywords: Internal limiting membrane peeling (ILM peeling); internal limiting membrane flap covering (ILM flap covering); vitrectomy; macular hole (MH); meta-analysis

Submitted Mar 20, 2021. Accepted for publication May 06, 2021.

doi: 10.21037/apm-21-871

View this article at: http://dx.doi.org/10.21037/apm-21-871 


\section{Introduction}

A macular hole $(\mathrm{MH})$ refers to a macular area neuroepithelial defect of the retina. Idiopathic macular hole (IMH) is the most common form of all MHs, accounting for approximately $83 \%$ (1), and refers to MHs that occur in the absence of associated primary diseases of the eye such as trauma and vitreoretinopathy (2). It mainly causes clinical manifestations such as decreased visual acuity and visual deformation. While mainly occurring in people older than 65 years, it affects $0.1-0.8 \%$ of adults over 40 years of age (3). About two-thirds of IMH patients are female, and $80 \%$ of them have monocular disease (4,5). The most common treatments for IMH are pars plana vitrectomy (PPV, known simply as vitrectomy), posterior vitreous detachment, internal limiting membrane (ILM) peeling, and gas filling of the vitreous cavity (6). In most MH surgeries, ILM peeling has become an essential step. The combination of PPV with ILM peeling is a very safe and effective procedure that can achieve MH closure in up to $98 \%$ of cases (7). The ILM flap coverage is an effective surgical technique for large, full-thickness idiopathic MHs and myopic MHs treatment. It was first reported in 1999 that the MH closure rate can be improved by the application of ILM peeling, after which Michalewska et al. demonstrated that refractory large MHs can be closed using ILM flap coverage (8). In recent years, the optimal surgical approach for the treatment of IMH has been controversial, with some papers supporting ILM flap coverage $(9,10)$, and others arguing that ILM flap coverage is not different from traditional ILM peeling for the treatment of IMH $(11,12)$. Despite the high rate of anatomical closure in both procedures, there is no consensus on whether the anatomical efficacy and functional outcome of internal limiting flap coverage is more favorable than that of complete stripping of the ILM technique. In this study, we comprehensively and quantitatively compared the advantages and disadvantages of PPV combined with ILM peeling and PPV combined with ILM flap coverage in IMH treatment and explored the optimal surgical method for the treatment of large $\mathrm{MH}$.

We present the following article in accordance with the PRISMA reporting checklist (available at http://dx.doi. org/10.21037/apm-21-871).

\section{Methods}

\section{Literature search methods}

Searching was conducted within PubMed, Web of
Science, Embase, CNKI, and Wanfang databases with the search terms "Inverted internal Limiting membrane flap technique" OR "Inverted ILM flap technique" AND "Internal limiting membrane peeling" OR "ILM peeling" OR "Internal limiting membrane removal" OR "Removing the ILM" OR "ILM peel" AND "idiopathic macular hole" OR "IMH". The search terms "vitrectomy, internal limiting membrane peeling, internal limiting membrane avulsion, internal limiting membrane flap coverage, idiopathic macular hole" were searched in Chinese databases such as China National Knowledge Infrastructure (CNKI) and Wanfang, and the possible selected articles were screened by a second search of the references of relevant articles, as well as conference papers and abstract articles. The search time was set from 2010 to 2020.

\section{Inclusion and exclusion criteria of literatures}

According to the participants, intervention measures, comparison, results, and study design (PICOS) protocol, we used the following criteria: (I) participants (participants): diagnosed with $\mathrm{IMH}$ and a minimum diameter of the hole $>400 \mu \mathrm{m}$; (II) Intervention measures (control, participants): the experimental group: participants chose PPV combined with ILM flap coverage, the control group: participants chose PPV combined with ILM peeling; (III) outcome measures: including the effective rate of $\mathrm{MH}$ closure [according to optical coherence tomography (OCT) detection], preoperative and postoperative best corrected visual acuity (BCVA); (IV) study design: all clinical studies on the efficacy of PPV combined with ILM peeling and PPV combined with ILM flap coverage in IMH treatment. The exclusion criteria were as follows: (I) combination with severe cataract, glaucoma, myopia, retinal detachment, ocular inflammation, and a history of ocular surgery; (II) follow-up time less than 1 month; (III) did not provide the data required for this meta-analysis, and did not yield such information upon request, as well as the original text of the literature obtained by the method; (IV) poor quality of literature, missing data, duplicate reports; (V) case reports, systematic reviews, and animal experiments.

\section{Literature data extraction}

Literature evaluation and data extraction were independently performed by 2 investigators, who screened the articles according to the inclusion and exclusion criteria, and discussed and negotiated with a third researcher in case 


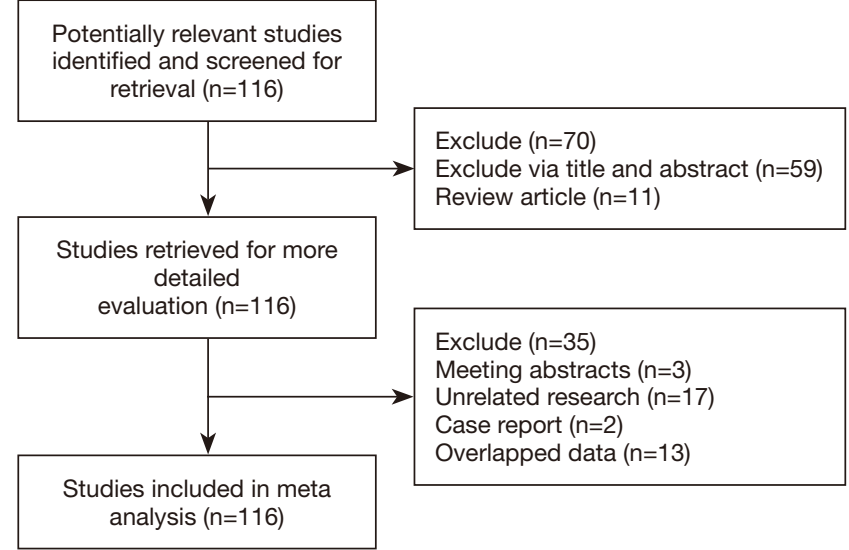

Figure 1 Characteristics of included literatures.

of data extraction disagreement. Data extraction content included: first author, publication year, country, real sample size of trial combination control, follow-up time, and so on.

\section{Statistical analysis}

Statistical analysis was performed using Stata16.0 software (StataCorp. LLC, College Station, TX, USA). Cochrane $\mathrm{q}$ test and $\mathrm{I}^{2}$ statistics were used to assess heterogeneity of included studies. The random-effects model was utilized when $\mathrm{P}<0.05$ and $\mathrm{I}^{2}>50 \%$, for meta-analysis. If this was not the case, the fixed-effects model was applied. Dichotomous variable data results were stated as odds ratio $(\mathrm{OR})$ with a $95 \%$ confidence interval $(\mathrm{CI})$, of numerical variable data outcomes were recorded as standardized mean difference (SMD) and a 95\% CI. Differences were considered statistically significant at $\mathrm{P}<0.05$. Sensitivity analysis was used to verify whether the conclusion of the meta-analysis was robust and credible. Funnel plots, Begg' test, and Egger's test was applied to evaluate if results had potential publication bias, and the test level $\alpha$ was taken as 0.10 .

\section{Results}

\section{General information of the involved studies}

The included literature processed in this meta-analysis is shown in Figure 1. Initially, 116 articles were redeemed with 46 selected after excluding 70 unqualified articles based on the title, abstract, and full text. The full text was further read; 3 conference proceedings, 17 unrelated articles, 2 case reports, and 13 duplicate studies were excluded, and finally 11 studies met the meta-analysis selection criteria $(7,11-20)$.
Among them, there were 4 Chinese articles and 7 in languages other than Chinese, with a total of 667 patients and 667 affected eyes. Seven hundred and twenty-eight cases were present in the test group and 731 in the control group. The characteristics of the included articles are displayed in Table 1.

\section{Meta-analysis results}

\section{Effective rate of hiatal closure}

A sum of 11 studies made a comparison between the 2 groups on the effective rate of hiatal closure with combined data showing a small $\left(\mathrm{I}^{2}=0.00 \%, \mathrm{P}=0.552\right)$ heterogeneity in each study, using the fixed-effect model. Meta-analysis showed that $94.4 \%$ (286/303 eyes) in the test group (PPV combined with ILM flap coverage) and 85.8\% (313/364 eyes) in the control group (PPV combined with ILM peeling) were closed. A statistically significant difference was observed in the $\mathrm{MH}$ closure rate between the 2 groups. The MH closure rate in the test group was superior when compared to the control group (OR $=3.36,95 \% \mathrm{CI}$ : 1.88 6.01, $\mathrm{P}<0.001$ ) (Figure 2).

\section{Best corrected visual acuity pre- and post-surgery}

A total of 11 studies compared preoperative BCVA (converted to $\log$ MAR visual acuity for recording), and 11 studies compared postoperative BCVA, (converted to logMAR visual acuity for recording). After data merging, a great heterogeneity was revealed in preoperative BCVA $\left(\mathrm{I}^{2}=54.00 \%, \mathrm{P}=0.017\right)$ and postoperative $\mathrm{BCVA}\left(\mathrm{I}^{2}=89.10 \%\right.$, $\mathrm{P}<0.001)$ in each study; therefore, the random effects model was used. The results showed that an absence of significant difference was observed in preoperative BCVA between the control group (SMD $=-0.18,95 \%$ CI: -0.42 to 0.06 , $\mathrm{P}=0.149$ ) (Figure $3 A)$. The BCVA (SMD $=-0.91,95 \% \mathrm{CI}$ : -1.43 to $-0.40, \mathrm{P}=0.001$ ) in the test group was better when compared to the control group (Figure $3 B$ ).

\section{Sensitivity analysis}

Sensitivity analysis outcomes showed that following modification of the inclusion criteria, eliminating inferiority studies, taking away maximum weights and erasing minimum weights of literature. The shared outcomes of hiatal closure efficiency of excluded literature showed no alterations from the original collective results. Thus, low sensitivity was shown (Figure 4). Additionally, the combined preoperative and postoperative BCVA results of each article 


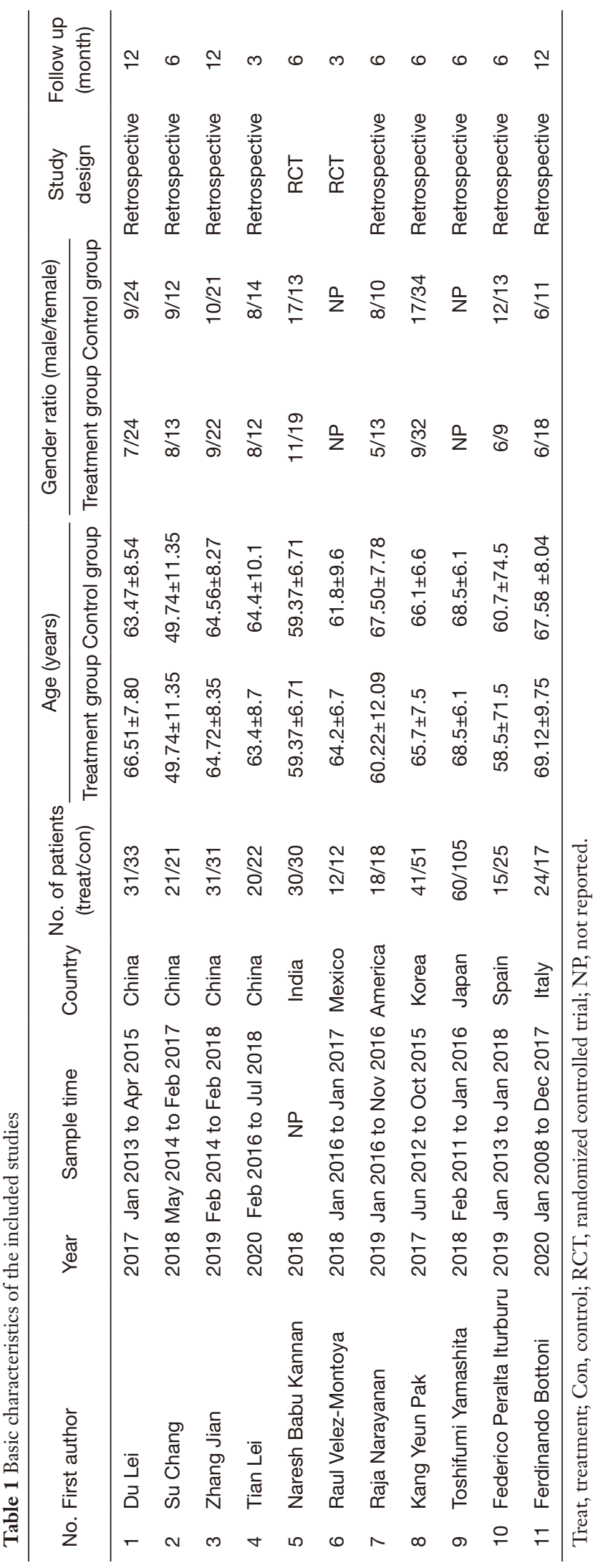

showed no alterations from the original combined results, with low sensitivity (Figure $5 A, B$ ). The outcomes established that the products of this meta-analysis were robust and credible.

\section{Publication bias}

The possibility of publication bias in the included articles was detected using funnel plots. In regards to the standardized error (SE, logOR) and $\log \mathrm{OR}$ of the effective rate of hiatal closure, the scatter diagram principally exhibited left-right symmetry about the symmetry axis with no significant publication bias in each study (Figure 6). The results of Begg's test $(\mathrm{Z}=0.93, \mathrm{P}=0.350)$ and Egger's test $(\mathrm{t}=1.00, \mathrm{P}=0.345)$ for closure effectiveness showed no publication bias. From the BCVA before and after surgery funnel plot, the scatter principally presented leftright symmetry about the axis of symmetry, indicating no significant publication bias present in each study (Figure $7 A, B)$. Further analysis showed that Begg's test $(\mathrm{Z}=0.62, \mathrm{P}=0.533)$ and Egger's test $(\mathrm{t}=-0.60, \mathrm{P}=0.563)$ were performed for preoperative BCVA, respectively, and the results showed that there was no publication bias. Begg's test $(\mathrm{Z}=2.02, \mathrm{P}=0.043<0.10)$ and Egger's test $(\mathrm{t}=-2.70$, $\mathrm{P}=0.025<0.10$ ) were performed for postoperative BCVA, respectively, and the results showed that there may have been some publication bias.

\section{Discussion}

The IMH is a common fundus macular disease. With the gradual deepening of knowledge about IMH and the continuous innovation and improvement of various treatment options, there have been gradual improvements in $\mathrm{MH}$ closure after IMH surgery. Patients with small MHs can be followed up for observation; for small and mediumsized $\mathrm{MH}$, intravitreal injection of Ocriplasmin has some clinical efficacy. However, for large MHs, surgical treatment is the only available option (21). However, there is still controversy surrounding the choice of treatment options for IMH, which need to be further explored. A sum of 11 studies was integrated into this meta-analysis, with similar study methods and high comparability. The visual acuity improvement of IMH was compared between the 2 groups to better guide the treatment of $\mathrm{MH}$.

Eleven studies in total made comparisons of the effective rate of hole closure between the 2 groups, and in almost all studies, the $\mathrm{MH}$ closure rate was high in 2 different surgical 


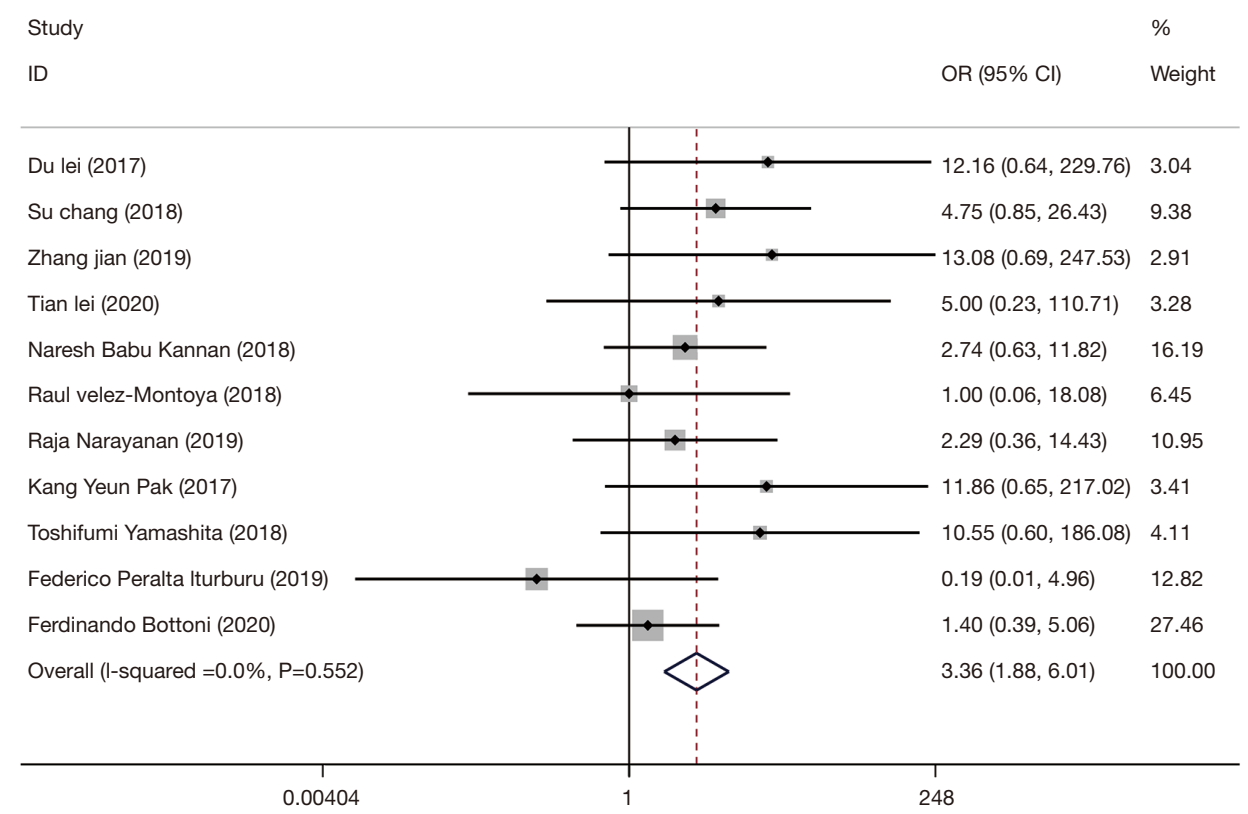

Figure 2 The effective rate of IMH closure in the 2 groups Forest map. IMH, idiopathic macular hole; OR, odds ratio; CI, confidence interval.

methods: PPV combined with ILM peeling and PPV combined with ILM flap coverage. Meta-analysis results showed a closure rate of $94.4 \%$ in the test group (PPV combined with ILM flap coverage) and $85.8 \%$ in the control group (PPV combined with ILM peeling). Test group $\mathrm{MH}$ closure rate was superior to the control group $(\mathrm{OR}=3.36$, 95\% CI: 1.88-6.01, $\mathrm{P}<0.001)$. The possible mechanism for the higher closure rate of MHs with ILM coverage is that ILM peeling can first completely remove the cortical vitreous, release macular traction, and stretch the retina, which could result in IMHs closure promotion and retinal reattachment (22). On this basis, ILM covering can further induce glial cell proliferation, resulting in MHs being filled with proliferating cells, thereby strengthening the degree of closure, can greatly improve the closure rate, and allow photoreceptors to reattach and localize directly close to the fovea (8), so ILM covering has a better closure effect.

11 studies in total additionally assessed the preoperative and postoperative BCVA. The results exhibited an absence of significant variation in the preoperative BCVA between the test group and control group (SMD $=-0.18,95 \% \mathrm{CI}$ : -0.42 to $0.06, \mathrm{P}=0.149$ ), while the postoperative $\mathrm{BCVA}$ in the test group (SMD $=-0.91,95 \%$ CI: -1.43 to -0.40 , $\mathrm{P}=0.001)$ was superior to that in the control group. The lack of difference in BCVA between the 2 groups before surgery indicated that the baseline levels of the 2 groups were well-balanced and comparable. The possible reason for the difference in BCVA between the 2 groups after surgery could be that according to the relevant literature, during the surgical operation, the retinal surgeon used the treatment method of ILM peeling "the more the better", resulting in extensive ILM peeling after surgery. Excessive ILM peeling predisposes to anatomical changes during follow-up, such as progressive separation of the optic nerve fiber layer, reduced macular papilla distance, and macular asymmetric displacement (23). Conversely, MHs closed after conventional ILM peeling are more likely to present a V-shaped, W-shaped, or flat/open closed pattern. Although they are good closure patterns, they may result in persistent loss of the photoreceptor layer (irregularities), retinal pigment epithelial defects, and loss of foveal tissue, which may be associated with poor visual recovery and the need for reoperation (24). In addition, there have been many reports in the literature on the relationship between postoperative $\mathrm{MH}$ closure and the occurrence of complications in patients with IMH. Modi et al. found that patients had already been impacted by irreversible damage to foveal photoreceptor cells, such as chorioretinal atrophy and staphyloma, before primary PPV (25). Ganglionic cells and the inner plexiform layer are the most vulnerable layers to manipulation and 


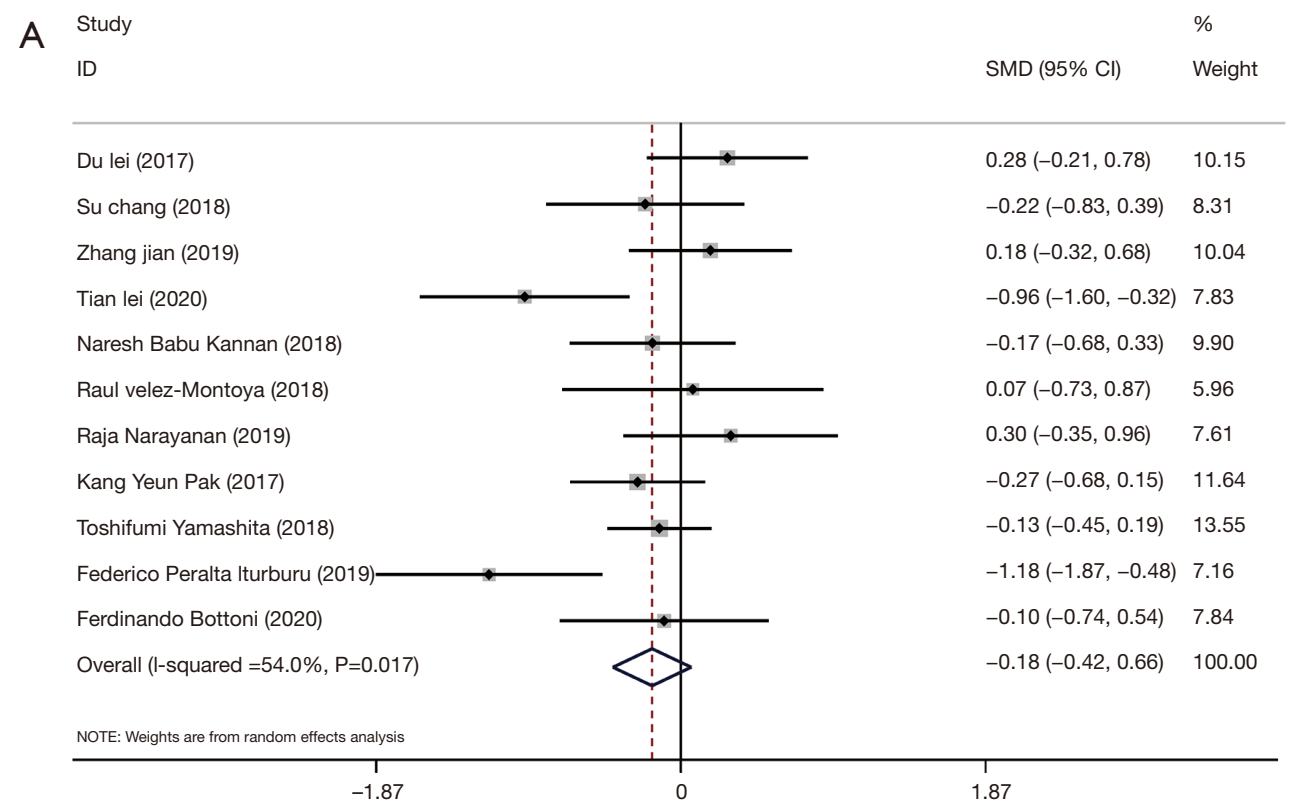

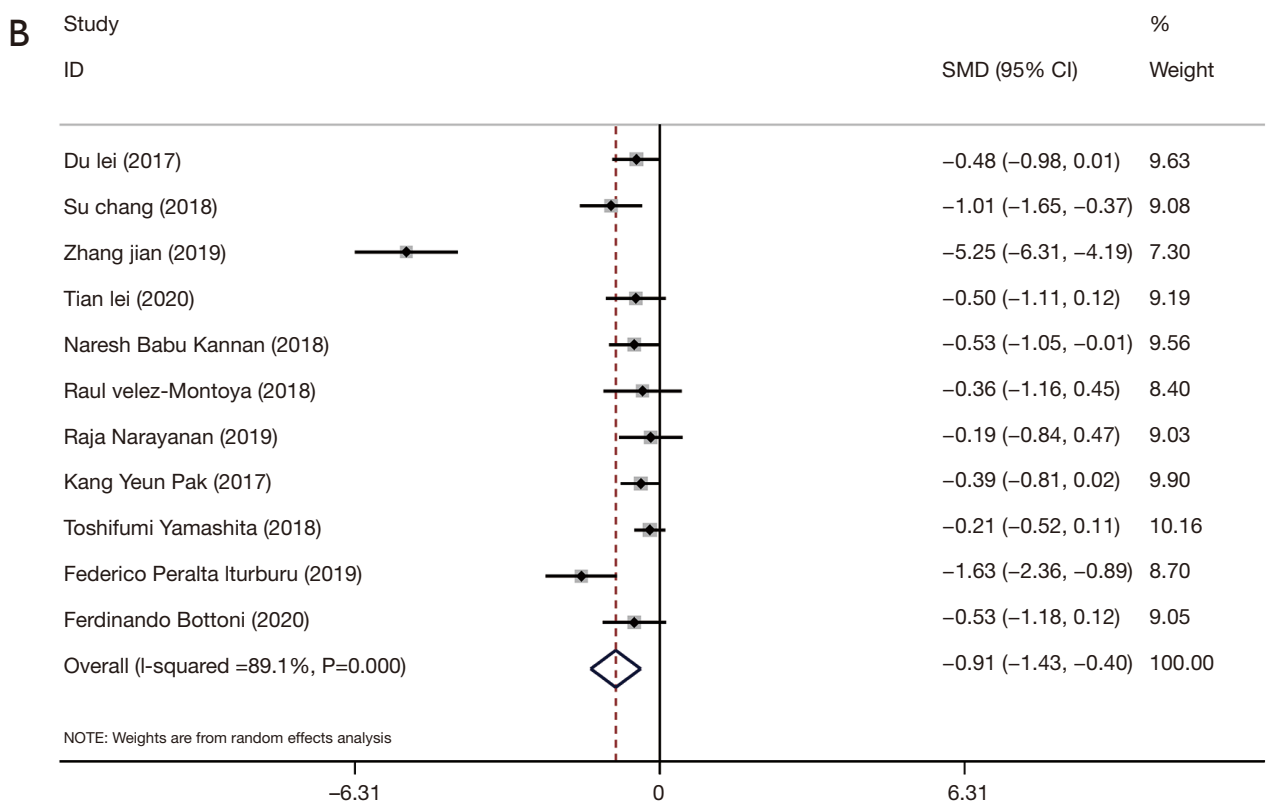

Figure 3 Forest map of BCVA before and after surgery. (A) Forest map of BCVA before surgery; (B) Forest map of BCVA after surgery. BCVA, best corrected visual acuity. 


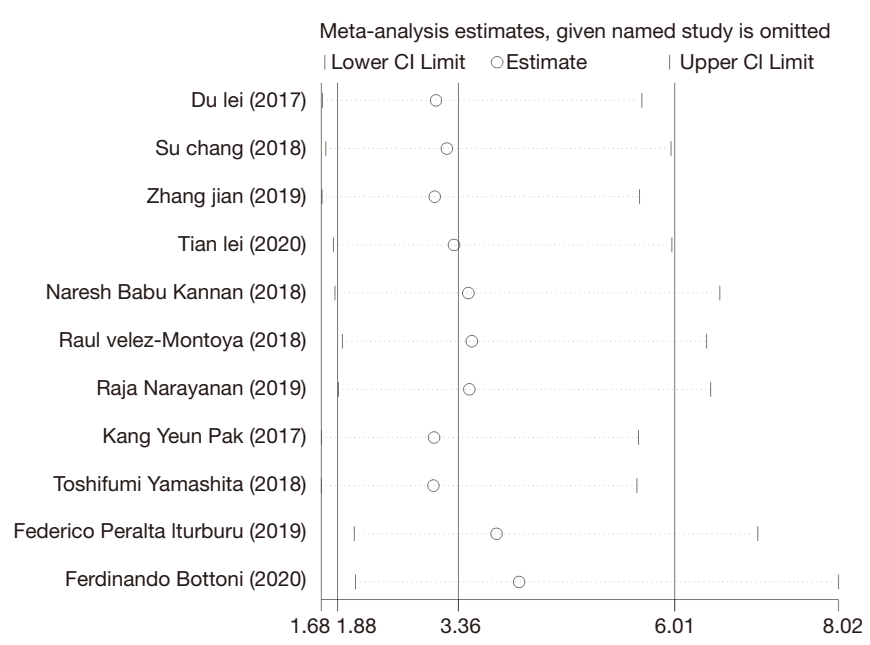

Figure 4 Sensitivity analysis of the effective rate of IMH closure in 2 groups. CI, confidence interval; IMH, idiopathic macular hole.
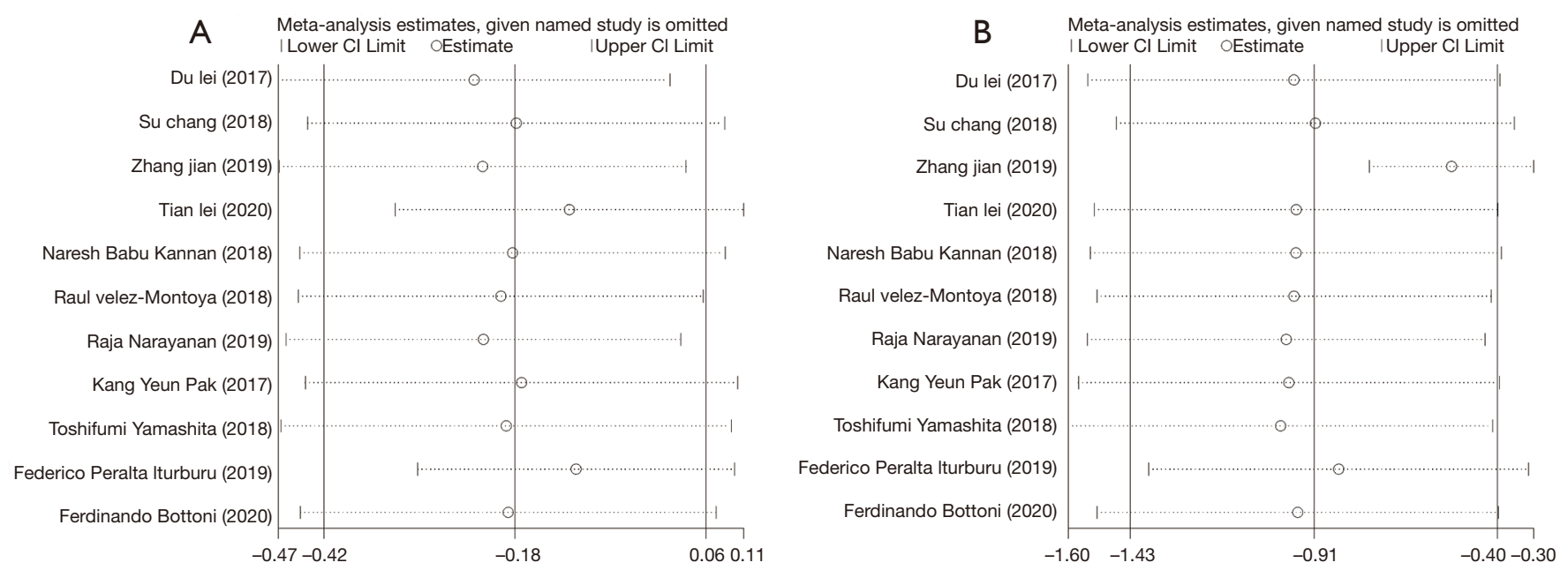

Figure 5 Sensitivity analysis of preoperative and postoperative BCVA. (A) Sensitivity analysis of preoperative BCVA; (B) Sensitivity analysis of postoperative BCVA. BCVA, best corrected visual acuity.

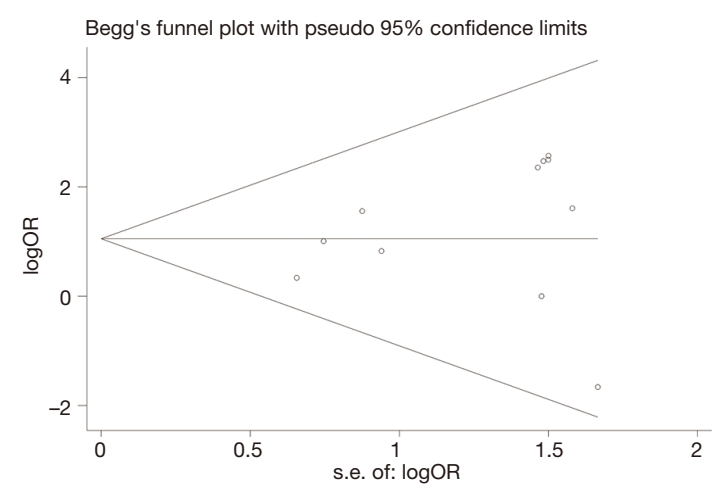

Figure 6 Funnel plot of the effective rate of IMH closure in the 2 groups. IMH, idiopathic macular hole. show significant damage in large areas around the fovea due to endarterectomy due to them being the ones that solely show thinning in the medial and temporal regions. Hayashi et al. found that the foveal photoreceptor layer has a possibility of being long destroyed and could not be recovered during ILM coverage, although the retina was reattached by coverage after surgery (26).

Of course, this meta-analysis still had some limitations. First, these studies had small sample sizes and insufficient follow-up times, which may have resulted in certain selection and information biases. Second, due to insufficient information obtained, the effects of various confounding 

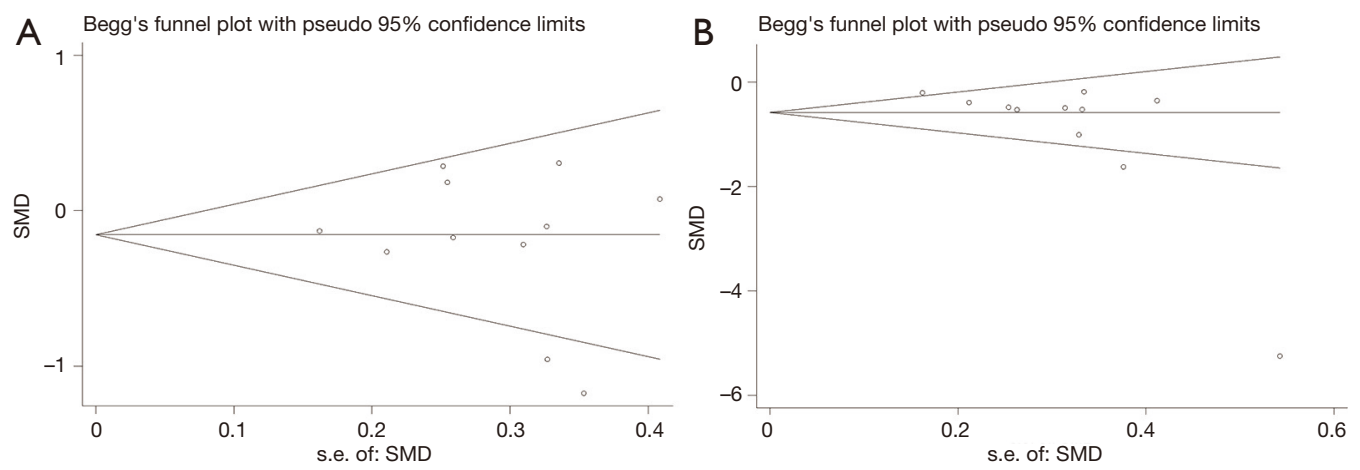

Figure 7 Funnel plot of BCVA before and after surgery. (A) Funnel plot of BCVA preoperatively; (B) funnel plot of BCVA postoperatively. BCVA, best corrected visual acuity.

factors such as age, stains, and intravitreal filling substances could not be analyzed. In the analysis, various studies tried to unify the baseline and correct the influence of age, gender, and other factors if it was possible. Finally, MHs of different grades may have corresponding optimal treatments, but we did not perform a detailed classification analysis of the severity of large holes.

In summary, the results of this meta-analysis showed that compared with traditional PPV combined with ILM peeling, PPV combined with ILM covering can significantly improve the $\mathrm{MH}$ closure rate and postoperative BCVA, while improving the functional effect and anatomical effect of IMH, with clinical application value.

\section{Acknowledgments}

Funding: This study was supported by the Traditional Chinese Medicine Science and Technology Program of Zhejiang Province of China (2020ZB138).

\section{Footnote}

Reporting Checklist: The authors have completed the PRISMA reporting checklist. Available at http://dx.doi. org/10.21037/apm-21-871

Conflicts of Interest: All authors have completed the ICMJE uniform disclosure form (available at http://dx.doi. org/10.21037/apm-21-871). The authors have no conflicts of interest to declare.

Ethical Statement: The authors are accountable for all aspects of the work in ensuring that questions related to the accuracy or integrity of any part of the work are appropriately investigated and resolved.

Open Access Statement: This is an Open Access article distributed in accordance with the Creative Commons Attribution-NonCommercial-NoDerivs 4.0 International License (CC BY-NC-ND 4.0), which permits the noncommercial replication and distribution of the article with the strict proviso that no changes or edits are made and the original work is properly cited (including links to both the formal publication through the relevant DOI and the license). See: https://creativecommons.org/licenses/by-nc-nd/4.0/.

\section{References}

1. Wang Y. Surgical Outcomes of Idiopathic Large Macular Hole after Limiting Membrane Flap Technique and Internal Limiting Membrane Peel: A Meta-Analysis. Medical University Of Fujian, 2019. Available online: http://www.doc88.com/p-04661880054002.html

2. Wu P, Wang X. Progress in idiopathic macular hole. International Journal of Ophthalmology 2014;2:259-62.

3. McCannel CA, Ensminger JL, Diehl NN, et al. Population-based incidence of macular holes. Ophthalmology 2009;116:1366-9.

4. Thapa SS, Thapa R, Paudyal I, et al. Prevalence and pattern of vitreo-retinal diseases in Nepal: the Bhaktapur glaucoma study. BMC Ophthalmol 2013;13:9.

5. Meuer SM, Myers CE, Klein BE, et al. The epidemiology of vitreoretinal interface abnormalities as detected by spectral-domain optical coherence tomography: the beaver dam eye study. Ophthalmology 2015;122:787-95.

6. Oh H. Idiopathic macular hole. Dev Ophthalmol 2014;54:150-8.

7. Velez-Montoya R, Ramirez-Estudillo JA, Sjoholm-Gomez 
de Liano C, et al. Inverted ILM flap, free ILM flap and conventional ILM peeling for large macular holes. Int J Retina Vitreous 2018;4:8.

8. Michalewska Z, Michalewski J, Adelman RA, et al. Inverted internal limiting membrane flap technique for large macular holes. Ophthalmology 2010;117:2018-25.

9. Rizzo S, Tartaro R, Barca F, et al. Internal limiting membrane peeling versus inverted flap technique for treatment of fullthickness macular holes: a comparative study in a large series of patients. Retina 2018;38 Suppl 1:S73-8.

10. Gu C, Qiu Q. Inverted internal limiting membrane flap technique for large macular holes: a systematic review and single-arm meta-analysis. Graefes Arch Clin Exp Ophthalmol 2018;256:1041-9.

11. Kannan NB, Kohli P, Parida H, et al. Comparative study of inverted internal limiting membrane (ILM) flap and ILM peeling technique in large macular holes: a randomizedcontrol trial. BMC Ophthalmol 2018;18:177.

12. Narayanan R, Singh SR, Taylor S, et al. Surgical outcomes after inverted internal limiting membrane flap versus conventional peeling for very large macular holes. Retina 2019;39:1465-9.

13. Du L, Chen Jia, Long T, et al. Effects of PPV combined with internal limiting membrane flap surgery on idiopathic macular hole. Recent Advances in Ophthalmology 2017;37:140-142, 146.

14. Peralta Iturburu F, Garcia-Arumi C, Bové Alvarez M, et al. Comparison of Anatomical and Visual Outcomes between Idiopathic and Myopic Macular Holes Using the Internal Limiting Membrane or Inverted Internal Limiting Membrane Flap Technique. J Ophthalmol 2019;2019:6723824.

15. Bottoni F, Parrulli S, Mete M, et al. Large Idiopathic Macular Hole Surgery: Remodelling of Outer Retinal Layers after Traditional Internal Limiting Membrane Peeling or Inverted Flap Technique. Ophthalmologica 2020;243:334-41.

16. Pak KY, Park JY, Park SW, et al. Efficacy of the PerfluoroN-Octane-Assisted Single-Layered Inverted Internal Limiting Membrane Flap Technique for Large Macular Holes. Ophthalmologica 2017;238:133-8.

17. Su C, Li W, Su RF, et al. Efficacy Analysis of Two Different Treatments for Idiopathic Macular Hole. Hebei Medical Journal 2018;24:973-6.

18. Tian L, Liu J, Lu WL. Efficacy analysis on vitrectomy combined with internal limiting membrane flap inversion comparing with vitrectomy combined with conventional internal limiting membrane peeling for treatment of macular hole. Recent Advances in Ophthalmology 2020;40:272-4.

19. Yamashita T, Sakamoto T, Terasaki H, et al. Best surgical technique and outcomes for large macular holes: retrospective multicentre study in Japan. Acta Ophthalmol 2018;96:e904-10.

20. Zhang J, Guan XD, Fang TM, et al. Observation on the Effect of Vitrectomy Combined with Internal Limiting Membrane Flap in the Treatment of Large Diameter Idiopathic Macular Hole. Shandong Medical Journal 2019;59:76-8.

21. Liu L, Xiao LB, Yu DY, et al. Pathogenesis and Treatment of Idiopathic Macular Hole. International Journal of Ophthalmology 2020;20:1907-12.

22. Spiteri Cornish K, Lois N, Scott NW, et al. Vitrectomy with internal limiting membrane peeling versus no peeling for idiopathic full-thickness macular hole. Ophthalmology 2014;121:649-55.

23. Ito Y, Terasaki H, Takahashi A, et al. Dissociated optic nerve fiber layer appearance after internal limiting membrane peeling for idiopathic macular holes. Ophthalmology 2005;112:1415-20.

24. Michalewska Z, Michalewski J, Dulczewska-Cichecka $\mathrm{K}$, et al. Temporal inverted internal limiting membrane flap technique versus classic inverted internal limiting membrane flap technique: A Comparative Study. Retina 2015;35:1844-50.

25. Modi A, Giridhar A, Gopalakrishnan M. Spectral domain optical coherence tomography-based microstructural analysis of retinal architecture post internal limiting membrane peeling for surgery of idiopathic macular hole repair. Retina 2017;37:291-8.

26. Yuan J, Zhang LL, Lu YJ, et al. Vitrectomy with internal limiting membrane peeling versus inverted internal limiting membrane flap technique for macular hole-induced retinal detachment: a systematic review of literature and meta-analysis. BMC Ophthalmol 2017;17:219.

(English Language Editor: J. Jones)

Cite this article as: Jiang T, Zhang L, Wan Q, Zhang Z. Comparative study of vitrectomy combined with internal limiting membrane peeling and vitrectomy combined with internal limiting membrane flap covering in idiopathic macular hole treatment: a meta-analysis and systematic review. Ann Palliat Med 2021;10(5):5474-5482. doi: 10.21037/apm-21-871 